\title{
Modeling of tokamak divertor plasma for weakly collisional parallel electron transport
}

\author{
M.V. Umanskya,1,*, A.M. Dimits ${ }^{\mathrm{a}}$, I.Joseph ${ }^{\mathrm{a}}$, J.T. Omotani ${ }^{\mathrm{b}}$, T.D.Rognlien ${ }^{\mathrm{a}}$ \\ ${ }^{a}$ Lawrence Livermore National Laboratory, Livermore, CA 94550, USA \\ ${ }^{b}$ Culham Laboratories, Culham Science Centre, Abingdon, Oxon OX14 3DB, UK
}

\begin{abstract}
The parallel electron heat transport in a weakly collisional regime can be represented in the framework of the Landau-fluid model (Hammett et al., Phys. Rev. Lett., 64, 3019, 1990). Practical implementation of Landaufluid transport has become possible due to the recent invention of an efficient non-spectral method for the non-local closure operators (Dimits et al., Phys. Plasmas 21, 055907 (2014)). Here the implementation of a Landau-fluid based model for the parallel plasma transport is described, and the model is tested for different collisionality regimes against Fokker-Planck simulations. The new method appears to represent the weakly collisional electron transport more accurately than the conventional flux-limiter based models, on the other hand it is computationally efficient enough to be incorporated in comprehensive edge plasma simulations.
\end{abstract}

Keywords: Parallel heat transport

${ }^{*}$ Corresponding author

Email address: umansky1@llnl.gov (M.V. Umansky)

${ }^{1}$ Mailing address: L-637, LLNL, 7000 East Ave., Livermore, CA 94550, USA

Preprint submitted to Journal of Nuclear Materials September 19, 2014 


\section{Introduction}

Tokamak edge plasma transport codes rely on a collisional fluid plasma model that is only accurate when the ion and electron collisional mean free paths are short compared to the spatial scales of the problem. This condition is at best marginally satisfied in present-day tokamak edge plasmas, and is likely to be violated in next-generation devices. In particular, departures from collisional Braginskii (Spitzer) parallel thermal conduction have a strong influence on the poloidal energy flux in the tokamak edge, especially in the electron channel, which poses a serious limitation on the utility of the existing transport models to weakly collisional edge plasmas. This raises demands for developing accurate and efficient representation of parallel transport within edge plasma modeling codes for the collisionless and weakly collisional regimes. Such a representation has been developed in the context of Landau-fluid (LF) models [1]. Practical implementation of LF parallel transport models in the framework of an edge modeling code has become possible due to the recent invention of an efficient non-spectral method for the non-local closure operators [2]. In this report, we describe implementation of a LF-based model for parallel electron transport. Results are presented for test problems in different collisionality regimes and tested against Fokker-Planck kinetic calculations. 


\section{Landau-fluid model}

The LF model for collisionless plasmas was originally proposed by Hammett and Perkins in their seminal paper [1]. The basic idea is to introduce closure terms in the fluid moment equations such that the resulting equations approximate the kinetic plasma linear response function. Here, we focus on the simplest model considered in [1], so-called $3+0$ model since it includes three parallel moment equations and none for the perpendicular moments:

$$
\begin{aligned}
& \frac{\partial}{\partial t} n+\frac{\partial}{\partial z}(u n)=0 \\
& \frac{\partial}{\partial t}(m n u)+\frac{\partial}{\partial z}(u m n u)=-\frac{\partial}{\partial z} p+e n E-\frac{\partial}{\partial z} S \\
& \frac{\partial}{\partial t} p+\frac{\partial}{\partial z}(u p)=-(\Gamma-1)(p+S) \frac{\partial}{\partial z} u-\frac{\partial}{\partial z} q
\end{aligned}
$$

Here $n$ is the fluid density, $u$ is the parallel velocity, $p$ is the pressure, and $q$ and $S$ are artificial terms providing the closure. Other symbols used are $e$ and $m$ for the electron mass and electric charge, $\Gamma$ is the adiabatic index, and $E$ is the electric field.

Equations (1-3) describe the parallel dynamics of electrons in plasma. The closure terms, represented in Fourier space, are the non-local heat flux,

$$
q_{k}=-n_{0} \chi_{1} \frac{\sqrt{2} V_{t}}{|k|} i k T_{k}
$$

and the non-local momentum flux

$$
S_{k}=-m n_{0} \mu_{1} \frac{\sqrt{2} V_{t}}{|k|} i k u_{k}
$$

Here $T$ is the temperature defined as $p / n, \chi_{1}$ and $\mu_{1}$ are the coefficients, and as described in [1], the choice

$$
\begin{array}{r}
\chi_{1}=2 / \sqrt{\pi} \\
\mu_{1}=0 \\
\Gamma=3
\end{array}
$$

optimizes the accuracy of the approximation of the plasma linear response function in the collisionless limit. 
Beyond the collisionless LF model, we use the approach proposed in $[4,5]$, to include electron collisions within the framework of the LF model

$$
q_{k}=-n_{0} \chi_{1} \frac{\sqrt{2} V_{t}}{|k|+\kappa_{0}} i k T_{k}
$$

where $\kappa_{0}$ is the measure of reciprocal electron collision mean-free-path,

$$
\kappa_{0}=0.5\left(\nu_{e i} / V_{t}\right)
$$

The form in Eq. (9) has the property that in the collisionless limit it obviously reduces to Eq. (4), and in the strongly collisional regime it is close to the Braginskii heat conduction flux,

$$
q_{B} \approx-3.2 \frac{n_{0} V_{t}^{2}}{\nu_{e i}} \frac{\partial T}{\partial z}
$$

The $3+0$ model considered here is one of the most basic LF models. A more general LF model, using a larger number of moment equations, in particular incorporating friction and thermal force terms, will be presented in a separate future publication [3]. 


\section{Lorentzian based calculation}

The nonlocal Landau-fluid heat flux in Eq. (4) is most easily written in Fourier space, which is convenient for calculations with a spectral code. However, most edge plasma modeling tools are non-spectral due to complicated geometry and strong variations in plasma parameters near the boundaries. For practical application to edge plasma modeling problems it would be much more efficient and convenient to calculate the heat flux in configuration space.

A non-spectral method for calculating expressions of the form Eq. (4) was recently invented [2]. The key idea is to use the sum of Lorentzian functions to approximate the $\operatorname{sgn}(k)$ function. The computational cost scaling with the problem size is similar to that for the Fourier transform for a serial computation, but the non-spectral method has more efficient scaling in a parallel computing environment.

First, we rewrite the heat flux given in Eq. (9) in the form

$$
q_{k}=A \frac{k}{|k|+\kappa_{0}} i T_{k}
$$

where $A=1.6 n V_{t}, \kappa_{0}=0.5 / \lambda_{e}$. As a function of $k$, the operator of interest is

$$
f\left(k, \kappa_{0}\right)=\frac{k}{|k|+\kappa_{0}}=g(\xi)=\frac{\xi}{|\xi|+1},
$$

where $\xi=k / \kappa_{0}$

The function $g(\xi)$ can be represented by a sum of $N$ Lorentzians

$$
g(\xi)=\sum_{j=1}^{N} \frac{\alpha_{j} \xi}{\xi^{2}+\beta_{j}^{2}},
$$

where $\alpha_{j}, \beta_{j}$ are coefficients found by a numerical optimization procedure, using the constraint $\sum_{j=1}^{N} \alpha_{j} / \beta_{j}^{2}=1$ to match exactly the strongly collisional limit. Using a larger $\mathrm{N}$ allows improving the accuracy and the range of validity of the fit; but it increases the computational cost. For calculations in this paper we use $\mathrm{N}=3$, as detailed in the Appendix. This provides a reasonably good approximation, within $1 \%$, for the nonlocal kernel from the strongly collisional regime, $x \rightarrow 0$, to a weakly collisional regime, $x=2 k \lambda_{e} \sim$ $2 \lambda_{e} / L_{T}=10$, where $L_{T}$ is the temperature gradient scale length.

Thus, the heat flux is a sum of terms 


$$
q_{k}=A \sum_{j=1}^{N} \frac{\alpha_{j} \xi}{\xi^{2}+\beta_{j}^{2}} i T_{k}=\sum_{j=1}^{N} q_{k}^{j}
$$

where

$$
q_{k}^{j}=-A \frac{\alpha_{j}\left(k / \kappa_{0}\right)}{\left(k / \kappa_{0}\right)^{2}+\beta_{j}^{2}} i T_{k}
$$

Each term in the sum $q_{k}^{j}$ can be written in Fourier space as

$$
\left[\left(\frac{k}{\kappa_{0}}\right)^{2}+\beta_{j}^{2}\right] q_{k}^{j}=-A \alpha_{j} \frac{k}{\kappa_{0}} i T_{k}
$$

which can be converted to a 2nd order ODE in configuration space:

$$
\left[-\frac{1}{\kappa_{0}^{2}} \frac{\partial^{2}}{\partial z^{2}}+\beta_{j}^{2}\right] q^{j}(z)=-A \alpha_{j} \frac{1}{\kappa_{0}} \frac{\partial}{\partial z} T
$$

Each ODE can be easily solved numerically by finite-difference as it is converted to a tridiagonal matrix problem. Note that originally, in Eq. (12), $\kappa_{0}$ was assumed constant but in Eq. (18) one can use $\kappa_{0}(z)$ as a function of space, which does not make the numerical solution any more difficult. For slowly varying $\kappa_{0}(z)$ this can be interpreted as a WKB-like approximation, but even rapidly varying $\kappa_{0}(z)$ has a well-defined solution.

In summary, the procedure for calculation of the heat flux corresponding to given $T(z)$ and $n(z)$ is forming and solving the ODE Eq. (18) for each $\mathrm{j}=1 \ldots \mathrm{N}$, and summing up the results, $q(z)=\sum_{j=1}^{N} q^{j}(z)$. 


\section{Results}

For the model verification, benchmark calculations were carried out with a Fokker-Planck code [6] implementing the kinetic closures originally developed by Ji and Held [7]. This model uses a moment expansion approach to solve a 1D kinetic equation including the full linearized Fokker-Planck (FP) collision operator. The test setup uses a set of 1D profiles of plasma density and electron temperature, and the heat flux is found in parallel from the kinetic calculation, and from using our Landau-fluid procedure.

The conventional approach to dealing with weakly collisional transport uses flux-limiters, e.g, [8]

$$
q_{B F L}=q_{B} \frac{1}{1+\left(1 / \alpha_{e}\right)\left(\lambda_{e} / \lambda_{T}\right)}
$$

where $q_{B F L}$ is the flux-limited Braginskii heat flux, $q_{B}$ is the standard Braginskii collisional heat flux, $\lambda_{e}$ is the electron collisional mean-free-path, $\lambda_{T}$ is the relevant temperature scale length, and $\alpha_{e}$ is a numerical coefficient, commonly chosen in the range $0.15-0.30$. The flux-limiting factor reduces to unity for the collisional limit, $\lambda_{e} / \lambda_{T} \rightarrow 0$, and it bounds the heat flux by the "free-streaming flux" $n V_{t} T$.

In the presented here test problems the density profile $n(s)$ is set flat, $\mathrm{n}=$ const, and the electron temperature profile $T(s)$ is set Gaussian, centered in the middle of the domain $\mathrm{s} \in[0,100], T(s)=1+10 \exp \left(-((s-50) / 10)^{2}\right)$, where $s$ is in $\mathrm{cm}$, and $\mathrm{T}$ in $\mathrm{eV}$. Figures (1-2) show the calculated profiles of the electron heat flux from the Landau-fluid model and from the FP model, for two of the studied cases, with $n=10^{13} \mathrm{~cm}^{-3}$ and $n=10^{14} \mathrm{~cm}^{-3}$. The Braginskii heat flux without flux-limiting (Brag) and with flux-limiting (BFL), is also shown on the same plots for comparison. One can observe that in these test problems the LF results appear significantly more accurate than BFL, in terms of approximating the kinetic FP results. That was generally the case for a number of test problems we have considered, due to the space limitations only a few representative cases are shown in this paper.

As expected, at higher density (and higher collisionality) the heat flux becomes close to the collisional Braginskii result; however only for $\nu^{*} \gtrsim 10^{2}$ the kinetic corrections become small (a few percent or less).

Based on our several benchmark test cases, using a single-peak Gaussian temperature profile $T(s)$ like those shown in Figs. (1-2), and others, with more complex forms of the $T(s)$ with multiple peaks (not shown here), the 
general observation is that for $\nu^{*}$ as small as $\sim 1$ the level of disagreement between the LF model and the FP solution is within a few percent, but for $\nu^{*} \sim 0.1$ the disagreement is already on the order of factor of 2 . On the other hand, the flux-limited Braginskii formula provides a few percent (or better) accuracy level only for $\nu^{*} \sim 10$ and above. Thus, based on the test problems that we have considered, compared to the flux-limited Braginskii, the proposed here LF model appears to be applicable to less collisional regimes. Figures (1-2) show that, for all the models considered here, the heat flux decays with $s$ for large $s$ where the temperature gradient is small. However, detailed analysis shows that in this region the LF and FP heat flux exceeds the collisional heat flux, which apparently is caused by weakly-collisional electrons bringing thermal energy from remote locations. Unlike the fluxlimiter expression, the nonlocal LF calculation allows for the heat flux to be nonzero where the local temperature gradient is zero, or even to go up the local temperature gradient. 


\section{Discussion}

Nonlocal fluid equations often arise in transport modeling when gradient scale is not large compared to the corresponding kinetic mean-free path. In this case, the number of particles contributing to a fluid moment flux at a given point in space depends not only on local thermodynamic condition but also on conditions at other spatial locations the particles can come from. Therefore the flux corresponding to a fluid moment is given by a convolution integral of the form [5]

$$
q(z)=\int g(z-\zeta) T(\zeta) d \zeta
$$

where $g$ is some kernel, which leads to time-evolution transport equations that are structurally integro-differential equations

$$
\partial_{t} f(x, t)=F\left(t, x, f, \partial_{x} f, \int K\left(x, x^{\prime}, t, t^{\prime}\right) f\left(x^{\prime}, t^{\prime}\right) d x^{\prime} d t^{\prime}\right)
$$

where $\mathrm{f}$ represents the state of fluid moments, and the right-hand side includes their spatial derivatives and spatial integrals.

Nonlocal fluid models have been extensively used for nonlocal electron thermal transport for ICF $[9,10,11,12,13,14]$, also for problems of nonlocal neutron transport [15] and radiation transport [16]. In the context of tokamak edge plasma, nonlocal fluid models have been considered in particular for the parallel electron heat flux [17, 18, 19, 20], and for neutral particle transport in plasma $[21,22,23]$.

Our LF heat flux calculation presented here is conceptually similar to this as it involves solving a set of ODEs along the parallel coordinate, which is equivalent to taking spatial integrals along the magnetic line. This is different from direct computation of the integral operator, e.g., in [9, 11, 12]. Unlike a heuristic integral heat flux operator, as, e.g., in [9, 11], our LF approach is closer to a first-principles theory.

Probably the accuracy of our method can be improved by performing more detailed comparisons against kinetic calculations and tuning up some of the model's parameters. In general, a numerical solution of integrodifferential equations can be an enormous computational task. However, for our LF nonlocal heat flux calculation using the Lorentzian representation of nonlocal kernels, the computational cost is not high as it only involves solving a few three-diagonal linear systems. Thus the new method should prove to 
be computationally efficient enough to be used in large-scale tokamak edge plasma simulations. 


\section{Conclusions}

The purpose of this study was to investigate the utility of using non-local Landau-fluid (LF) models [1] for the parallel electron heat transport in the weakly collisional regime. The LF model considered here has been tested against Fokker-Planck simulations over a range of collisionality regimes. The presented here analytic and computational results demonstrate that the parallel electron heat transport in the weakly collisional regime can be rather accurately represented in the framework of LF models. The LF model proposed here appears to be applicable to the regimes of significantly lower plasma collisionality, compared to the conventional flux-limiter based models. Moreover, the LF model captures important features of weakly collisional electron transport than cannot be captured by the flux-limiter based models. The discussed here implementation of LF models uses the recent idea of representing non-local closure operators by a sum of Lorentzians [2]. The new method appears to be computationally efficient which makes it promising for using in comprehensive tokamak edge plasma simulations. 


\section{Appendix}

Here we describe a nonlinear fit of function $y(x)=x /(x+1)$ by three Lorentzians

$$
y(x)=\alpha_{1} L\left(x, \beta_{1}\right)+\alpha_{2} L\left(x, \beta_{2}\right)+\alpha_{3} L\left(x, \beta_{3}\right)
$$

where

$$
L(x, \beta)=\frac{x}{x^{2}+\beta^{2}}
$$

The nonlinear fit to determine the six parameters $\alpha_{i}, \beta_{i}$ is carried out with the MPFIT algorithm [24], which allows approximating the function $y(x)$ within $1 \%$ accuracy in the range $x \in[0,10]$, see Fig. (3).

The resulting values are

$$
\begin{array}{rll}
\alpha_{1}=0.0018 & \alpha_{2}=0.0769 & \alpha_{3}=2.4498 \\
\beta_{1}=0.1192 & \beta_{2}=0.4913 & \beta_{3}=2.1495 .
\end{array}
$$

These values are used for the calculations presented in this report. 


\section{Acknowledgment}

This work was performed under the auspices of the U.S. Department of Energy by Lawrence Livermore National Laboratory under Contract DEAC52-07NA27344. The authors are grateful for useful discussions with Drs. B.D. Dudson, P.C. Stangeby, and X.Q. Xu. 


\section{References}

[1] G. W. Hammett and F. W. Perkins. Phys. Rev. Lett. 64, 3019 (1990).

[2] A. M. Dimits, I. Joseph, and M. V. Umansky, Phys. Plasmas 21, 055907 (2014).

[3] I. Joseph "Connecting the Landau-fluid plasma model to collisional limit" in preparation (2014).

[4] M. A. Beer and G. W. Hammett, Phys. Plasmas 3, 4046 (1996).

[5] P. B. Snyder, G. W. Hammett, and W. Dorland, Phys. Plasmas 4, 3974 (1997).

[6] J. T. Omotani and B. D. Dudson, Plasma Phys. Control. Fusion 55, 055009 (2013).

[7] J. Y. Ji et al., Phys. Plasmas 16, 022312 (2009).

[8] P. C. Stangeby "The plasma boundary of magnetic fusion devices", Institute of Physics Publishing, Bristol and Philadelphia, 2000.

[9] J.F. Luciani, P. Mora and J. Vermont, Phys. Rev. Lett. 51 (1983), p. 1664.

[10] Epperlein, E. M., Rickard, G. J., and Bell, A. R. Physical Review Letters 61, 2453-2456 (1988).

[11] Epperlein, E. M. and Short, R. W., Phys. Fluids B 3, 3092 (1991).

[12] Maximov, A. V. and Silin, V. P., Physics Letters A, v.173, 1, 25, p. 83 (1993).

[13] G. P. Schurtz, Ph. D. Nicola, and M. Busquet, Phys. Plasmas, Vol. 7, No. 10, (2000).

[14] A.V. Brantov, V.Yu. Bychenkov, V.T. Tikhonchuk, W. Rozmus, Phys. Plasmas 5 (1998), pp. 2742-2753

[15] D. L. Henderson and C. W. Maynard, Nucl. Sci. Eng, 1989, 102, 172.

[16] K. R. Olson and D. L. Henderson, Ann. Nucl. Energy, 2004, 31,1495. 
[17] Yu.L. Igitkhanov and P. Yushmanov, Contrib. Plasma Phys. 28 (1988) 341.

[18] Yu.L. Igitkhanov and A.Yu. Pigarov, J. Nucl. Mater., v. 176-177, 1990, pp. 557-561.

[19] S. Ohtsu, S. Tanaka and M. Yamawaki, J. Nucl. Mater., v. 220-222, 1995, pp. 1005- 1009.

[20] Rabinski, M., Contributions to Plasma Physics, v. 30, 1, pp. 121-126, 1990.

[21] K. Audenaerde et al. J. Comp. Phys. 34, 268 (1980).

[22] P. M. Valanju. J. Comp. Phys, 88:114, 1990.

[23] W. M. Stacey and J. Mandrekas. Nuclear Fusion, 34:1385, 1994.

[24] C. B. Markwardt, Astronomical Data Analysis Software and Systems XVIII, 411, 251 (2009). 


\section{Figure captions}

Figure 1:

Test case $\mathrm{n}=10^{13} \mathrm{~cm}^{-3}$. The profiles are shown only to the right of the midpoint $\mathrm{s}=50 \mathrm{~cm}$. The top plot shows the profiles of $T_{e}$ (solid), $\lambda_{T}$ (dotted), and $\lambda_{e}$ (dashed). The bottom plot shows the calculated parallel electron heat flux $q_{\| e}$ as labeled in the plot: Landau-fluid (LF, solid), Fokker-Planck (FP, dashed), Braginskii (Brag, dotted), and flux-limited Braginskii (BFL, shaded) for $\alpha_{e} \in[0.15,0.30]$.

\section{Figure 2:}

Test case $\mathrm{n}=10^{14} \mathrm{~cm}^{-3}$. The profiles are shown only to the right of the midpoint $\mathrm{s}=50 \mathrm{~cm}$. The top plot shows the profiles of $T_{e}$ (solid), $\lambda_{T}$ (dotted), and $\lambda_{e}$ (dashed). The bottom plot shows the calculated parallel electron heat flux $q_{\| e}$ as labeled in the plot: Landau-fluid (LF, solid), Fokker-Planck (FP, dashed), Braginskii (Brag, dotted), and flux-limited Braginskii (BFL, shaded) for $\alpha_{e} \in[0.15,0.30]$.

Figure 3:

The fit to the function $\mathrm{y}(\mathrm{x})=\mathrm{x} /(\mathrm{x}+1)$ by three Lorentzians. The cross symbols show $\mathrm{y}(\mathrm{x})$, the green line shows the fit, and the red lines show contributions from individual Lorentzian terms. Same data shown in log-log scale (top) and log-lin scale (bottom). 

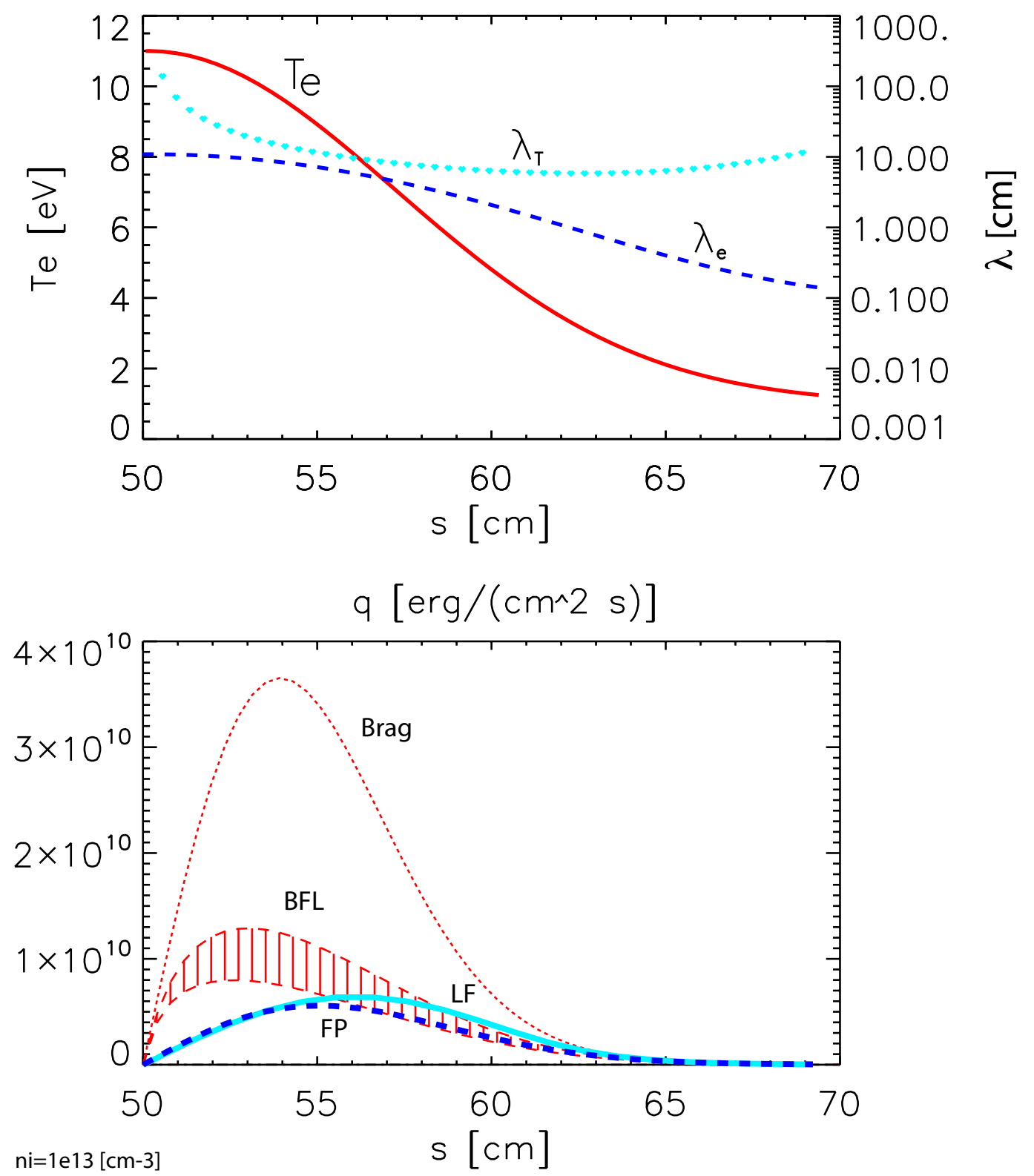

Figure 1: 

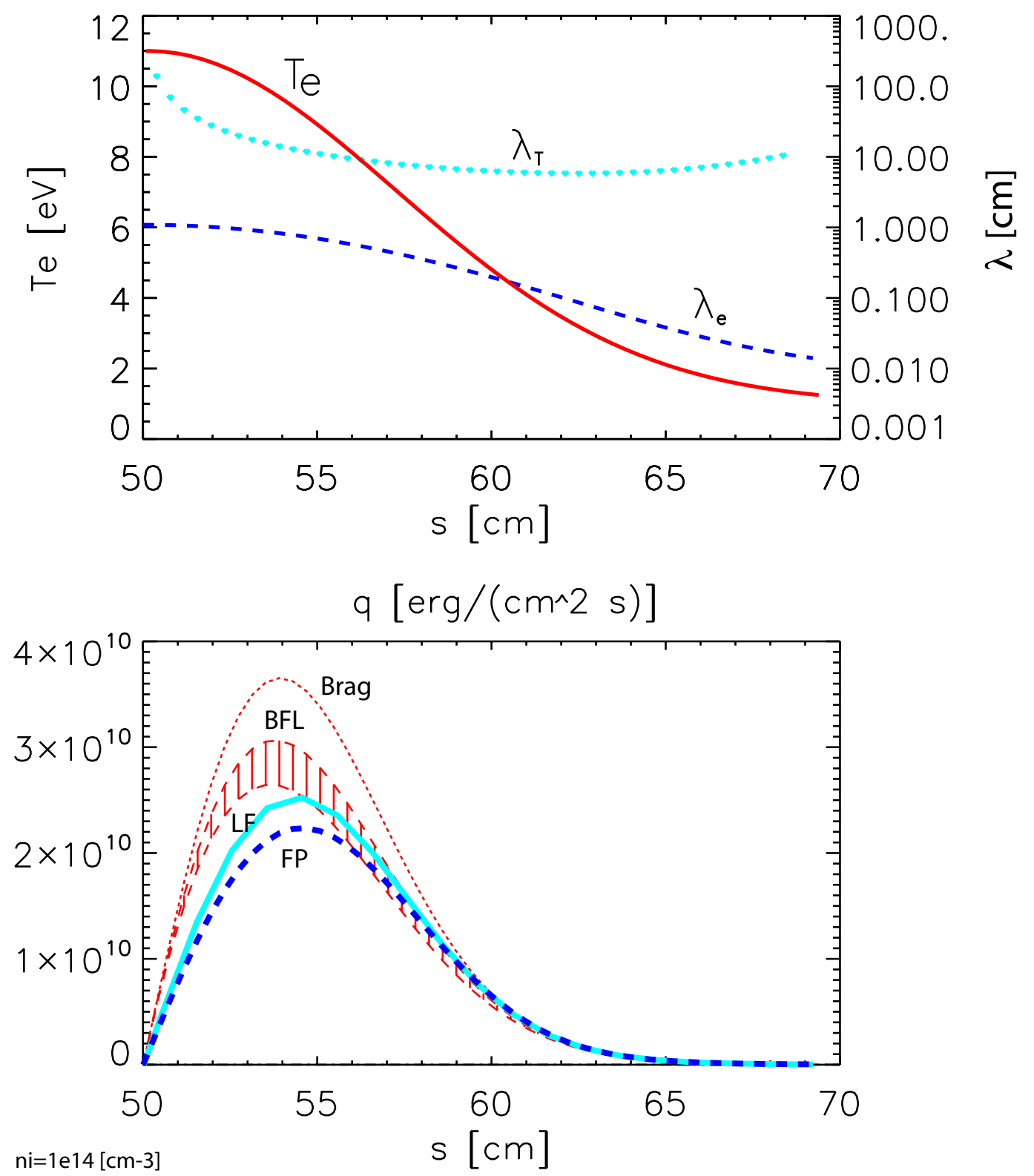

Figure 2: 
Fit to $y(x)=x /(x+1)$ by $x$ Lorentzions

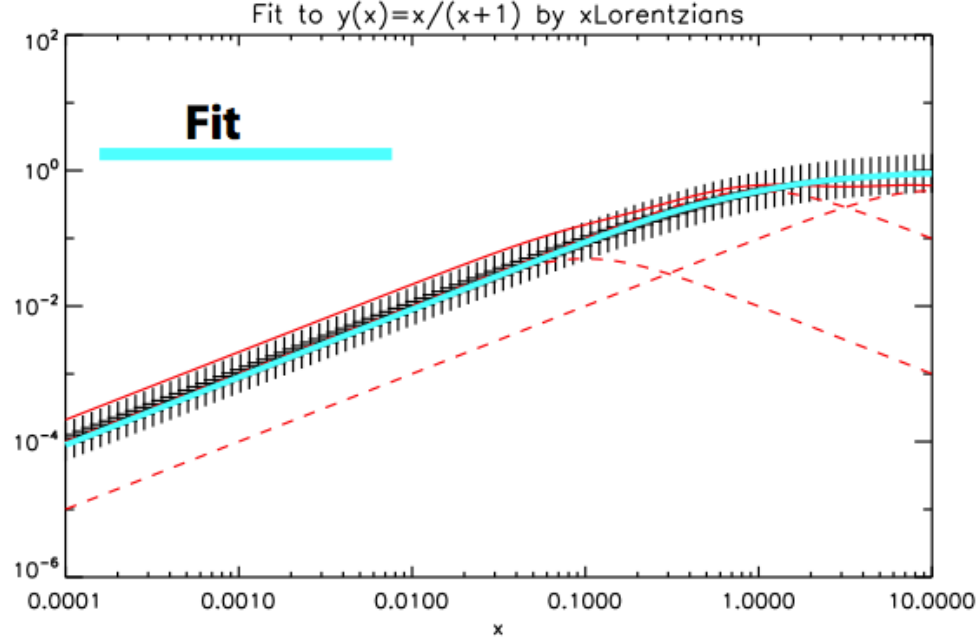

Fit to $y(x)=x /(x+1)$ by $x$ Lorentzions

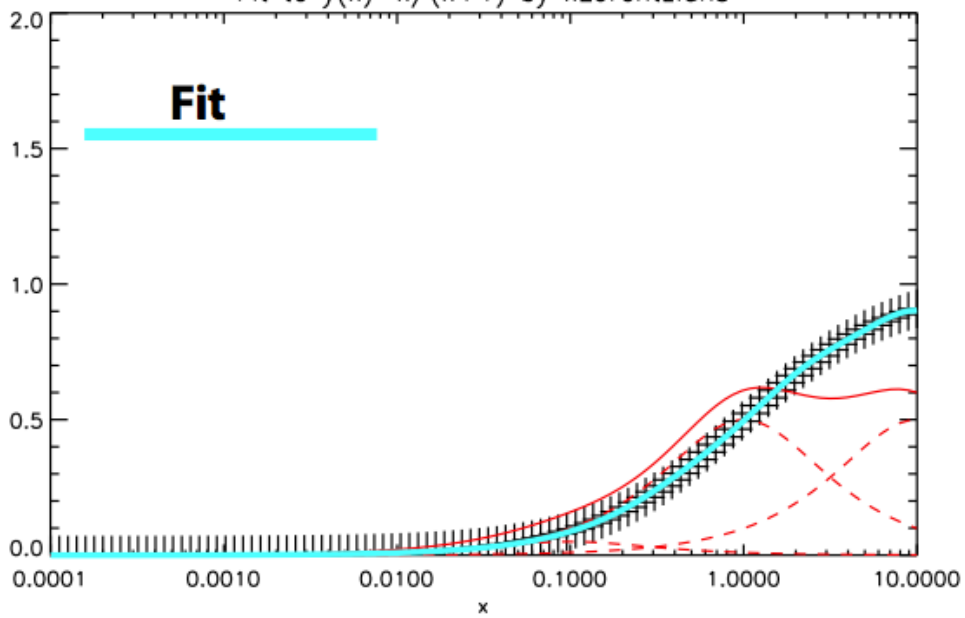

Figure 3: 